\title{
An Engineering Technology Course in Additive Manufacturing
}

\section{Prof. Christopher David LeBlanc, University of New Hampshire}

Christopher D. LeBlanc is currently the Program Coordinator and Assistant Professor for the Engineering Technology program at the University of New Hampshire Manchester campus. Prior to his faculty appointment he spent 16 years at International Business Machines (IBM) as an Analog Mixed Signal design engineer.

\section{Dr. Donald J. Plante, University of New Hampshire}

Donald J. Plante is a lecturer of Mathematics at the University of New Hampshire. His main area of research is in fractal geometry, although he also holds interests in 3D printing and mathematical modeling. 


\title{
An Engineering Technology Course in Additive Manufacturing
}

\begin{abstract}
The University of New Hampshire Manchester campus(UNHM) Department of Applied

Engineering and Sciences (ASE) Engineering Technology (ET) program has developed a course (ET401) investigating methods in additive manufacturing through the design and fabrication of 3D models. The curriculum is designed to have students gain skills by applying and integrating techniques from mathematics, engineering, and computing to develop 3D models that can be manufactured by commercially available 3D printers. The course is a four credit hour elective with no pre-requisites available to both ET and non-ET students. ET401 is intended to fulfill the environment, technology, and society (ETS) requirement of the UNHM Discovery program so particular emphasis is placed on ways 3D printing has effects on both the environment and the collective society. An early version of the course was offered at USS in the spring semester of 2016 with eight participating students. This work describes the structure of the course and methods used for assessment of the students.
\end{abstract}

Student evaluation was based on participation, discussion board activity, portfolio of weekly projects, and a final project. Weekly participation in an online discussion board was required to explore further impacts of 3D printing on the environment and society through its use in various disciplines. Students were tasked to identify areas in society likely to be impacted by new additive manufacturing techniques. Through discussion and development of the portfolios students were able to explore the effectiveness of current advances in 3D printing technologies and model complex geometric shapes using various computer software applications. The class portfolios are published under a creative common license making designs available to the public and for use in future semesters. Participants were encouraged to revise and improve upon their own work as well as other's existing models through the open source platform. For the final project students were required to propose a project that represented an original contribution to either engineering, art, or mathematics. Assessment of the final project was based on the following criteria: aesthetic appeal, functionality, durability, and complexity. After completion of the course participants demonstrated the ability to identify and define basic terms of additive manufacturing, and compare and contrast multiple manufacturing techniques impacts on the environment.

\section{Introduction}

The University of New Hampshire (UNH) of Manchester is the urban campus of the UNH system. UNHM offers ABET accredited Bachelor of Science (BS) degrees in Engineering Technology (ET). All students pursing a baccalaureate degree must complete the UNH Discovery requirements. "The Discovery Program provides the intellectual framework for students in any major. It represents the faculty's collective belief in what constitutes and contributes to essential knowledge of the world."1 The Discovery program covers studies in areas such as the foundations of English, Quantitative Reasoning, Biological Sciences, Physical Sciences, Fine and Performing Arts, Humanities, Historical Perspectives, World Cultures, Social Sciences, and Environment-Technology-Society (ETS). Most of the Discovery classes are 
offered from the College of Liberal Arts (COLA). Prior to this work the ET program had no classes that fulfilled any of the UNH Discovery requirements.

There is a lot of anecdotal and research evidence that deeply technical degrees such as ET can be isolating for students and lack diversity ${ }^{2}$. During advising periods many ET students are resistive of the course selection to satisfy the UNH Discovery program requirements. This can also be true for students pursing non-technical degrees; they can be resistive to taking classes with overly technically sounding course titles. The purpose of offering an ET course in additive manufacturing ( $3 \mathrm{D}$ printing) was to leverage the popularity of this emerging technology $\mathrm{y}^{3}$ to get ET students to work collaboratively with non-technical students in an effort to explore the impacts technology has on society. The hope is to get both populations of students to broaden their perspective on how technologies can impact the environment and society without the prerequisites of advanced mathematics or advanced engineering analysis.

In 2013 UNHM established the Engineering Computing Laboratory (ECL). Initially the ECL was intended to house the Senior Capstone project laboratory for Engineering and Computing Technology students. The 1,900 square foot facility was funded by a private foundation and has several workbenches outfitted with electronic work stations and light material fabrication equipment. In 2016 a 3D printer laboratory was added, currently there are eight 3D printers and a table top CNC machine, shown in Figure 1. Initially the facility was secured and only Engineering and Computer Technology students were allowed badge access to the space while they were enrolled in a capstone project class. As students, other faculty, and staff became aware of the space a lot of inquiry on use of the 3D printers was directed to the ET program. After a series of collaborative discussions with faculty in the Mathematics program the idea to offer a course in additive manufacturing was established. In the spirit of not having the course and facilities exclusive to students pursing technical degrees the curriculum was built to satisfy the Environment-Technology-Society Discovery program requirements. The hands on collaborative nature of the curriculum is only suited for small class sizes. The typical student to Faculty ratio at UNHM is around 11:1, for this class the enrollment was capped at 12 students. The class ET401 Introduction to 3D printing was first offered in the spring of 2017 with eight students enrolled, currently in the spring 2018 semester there are 13 students enrolled.

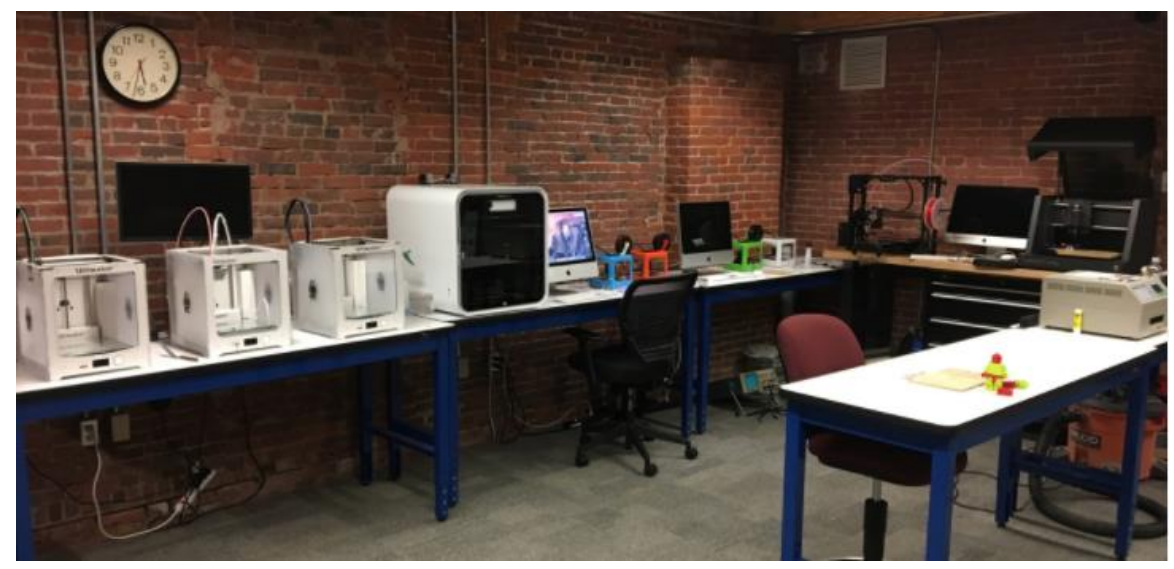

Figure 1 
The ET 3D printing lab consists of eight printers, two UltiMaker 2+, one UltiMaker 3, four micro M3D, one TAZ Lutzbot, one CubePro Duo, and one Carvy table top CNC machine. All models are single filament with the exception of the UltiMaker3 and CubPro Duo. Figure 2 shows the various models used in the class.

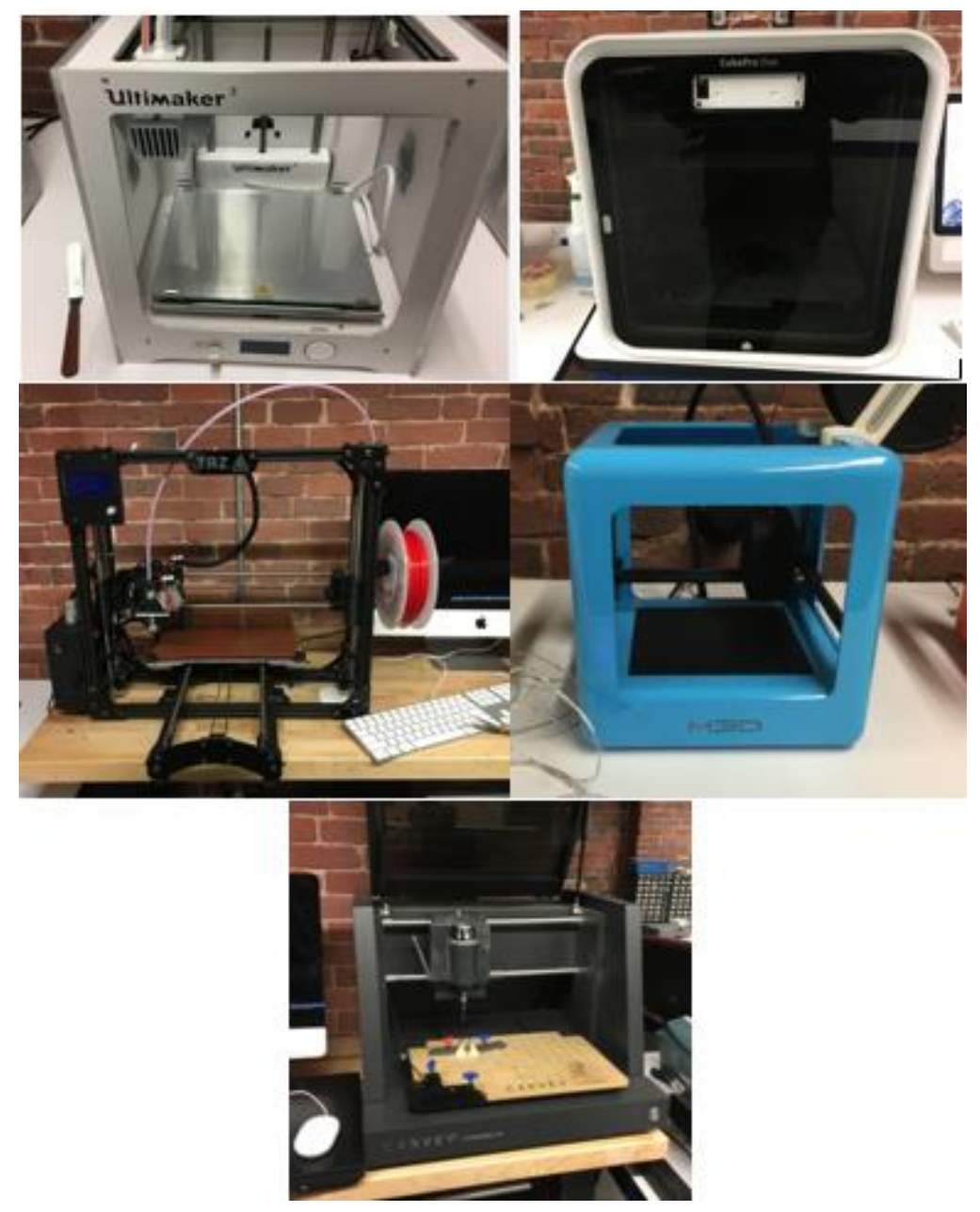

Figure 2

ET401 Learning Outcomes: After completion of this course students were able to:

- Identify and define basic terms in additive manufacturing

- Compare and contrast multiple additive manufacturing techniques impact on the environment

- Identify areas in society likely to be impacted by new additive manufacturing techniques

- Assess the effectiveness of current advances in 3D printing technologies

- Model complex geometric shapes using various computer software applications

- Revise and improve upon their own as well as other's existing models

- Publish their work under a creative common license as an open resource 


\section{ET401 Class Structure}

Participation: Attendance was mandatory, as was being punctual. Evaluating a class participation portion of a grade is always subjective and takes many things into account, such as being engaged while in class. The student's attitude drives so many important factors having to do with class participation (or lack thereof); e.g., do they come to class late (and if so, how often), do they have un-excused absences (and if so, how many), does their cell phone ring during class (or even worse, do they read or send text messages during class). Class participation policy was not intended to be punitive, but rather, to be a reward to show students how much value there is in coming to class. Coming to class on time, turning off their cell phones, not being disruptive, et cetera, is about showing respect for all participants, their peers, and the instructor.

Portfolio: The students in ET 401 documented each of their projects by making a design portfolio showing off their work. The portfolio gave a visual and textual representation of their progression through each project. The design portfolio acted as a resource for others to be able to learn from their work, and as such, its goal was to document the design process with enough detail so that others in society are easily able to recreate the designs. For this reason the portfolio was to be published under a creative common license and will be used in future semesters as a resource for students in the class.

Discussion Board: Each week during the semester begins with an in-class discussion on the environmental and societal impacts of the 3D printing techniques and technologies featured in that week's project. Students were required to participate in a weekly online discussion board where they were tasked with exploring further impacts of 3D printing on the environment and society through its use in various disciplines. Each post was required to identify at least one environmental and one societal impact for the topic under discussion. Students were required to post a meaningful reply to another classmate's initial post, where they compared and contrasted the impacts by providing additional examples.

Final Project: The final project in the class was to create and document a design of the student's choosing using all of the techniques that were learned throughout the semester. The design chosen needed to make an original contribution to engineering, art, or mathematics, and also needed to be approved by the instructor prior to implementation. The final designs were graded based on the following criteria: aesthetic appeal, functionality, durability, and complexity.

\section{ET410 Portfolio Projects}

- Penny Trap

- Working Hinge

- Reverse Engineering

- Modified Block

- Chess Set Team Challenge

- Fractal Geometry

- Metallic Pendant

- Dissolvable Support Required 
- Customizable Object

- Final project: Action Figure

\section{Portfolio Project Descriptions}

Penny Trap ${ }^{4}$ : This project guided students through the process of creating a penny trap. This unique design can only be constructed by the use of a Fused Deposition Modeling (FDM) 3D printer as it traps the penny inside of a cube one layer at a time. This project, an example shown in Figure 3, helped to illustrate the differences between traditional manufacturing techniques and what is possible with the advent of the $3 \mathrm{D}$ printer.

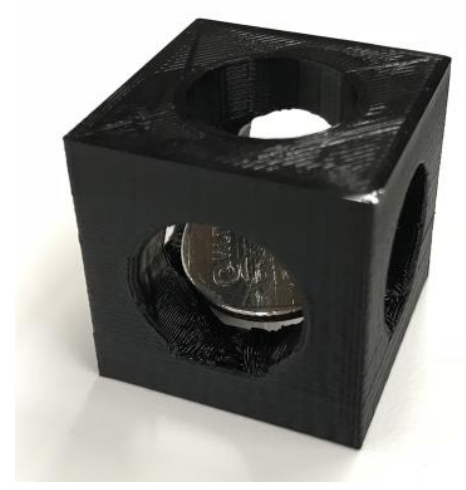

Figure 3

Working Hinge: The hinge is a key component in being able to construct mechanical machines that consist of multiple moving parts. Engineers design compound machines to make tasks easier and to benefit society. This lesson encouraged students to think about past machine inventions and their usefulness in our lives. For this project a working hinge or lever was designed and tested. An example of this project can be seen in Figure 4.
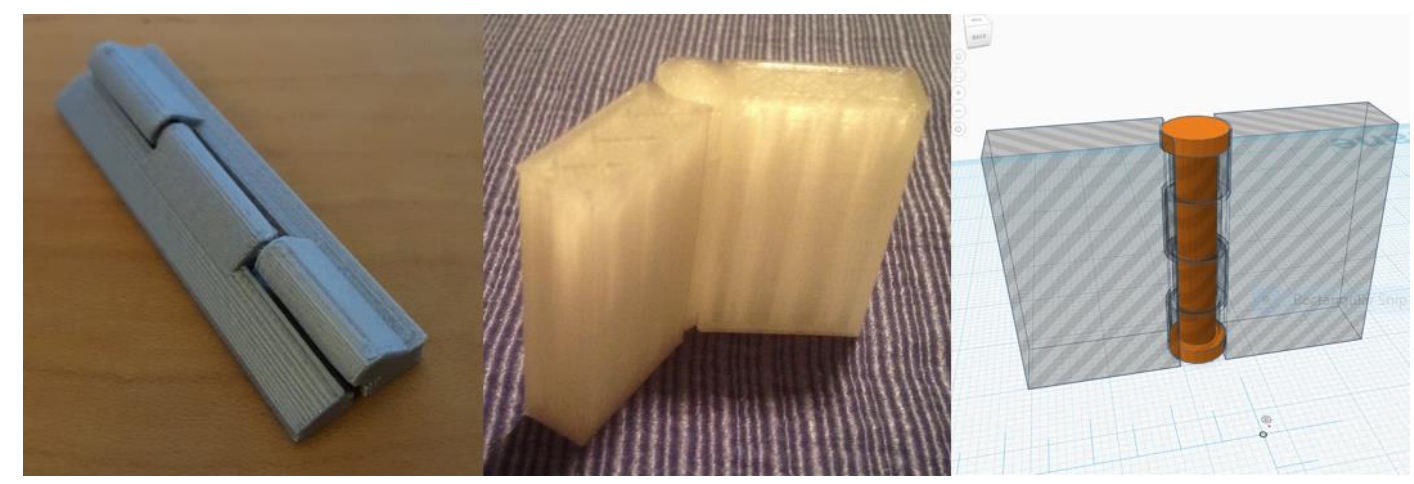

Figure 4 
Reverse Engineering: Reverse engineering is a critical skill that allowed students to learn how something works by first taking it apart. The practice is applicable, not only in the field of engineering, but also in computer science and biology, etc. A programmer might take apart the computer code of a virus or a biologist might sequence your DNA. For this project the students reverse engineered a children's play block with the specification that their copy must fit together perfectly with the original. The lesson explored the tolerance levels of current 3D printing technology to the student. The project as shown in Figure 5 was to reverse engineer a Lego Duplo block.

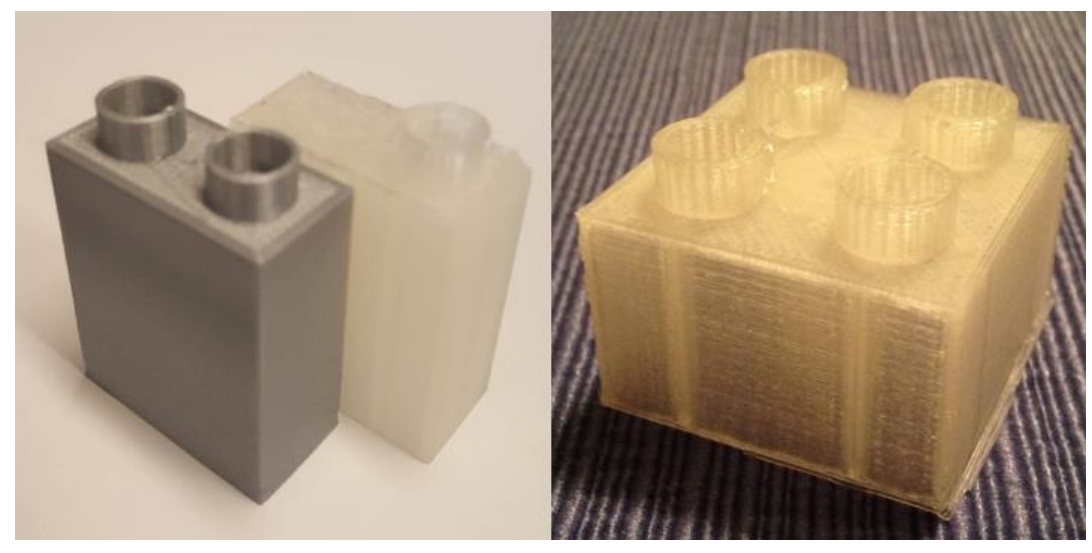

Figure 5

Modified Block: In continuation of the reverse engineering project students further improved the block that was designed by enhancing it in a new and original way. Students were free to modify their design in any way that they liked as long as it provided at least one new functionality to the original block set. Two examples of this are seen in Figure 6. The image on the left shows a rotating Duplo block, and the image on the right shows a phone case that can attach to a stand made from Legos.

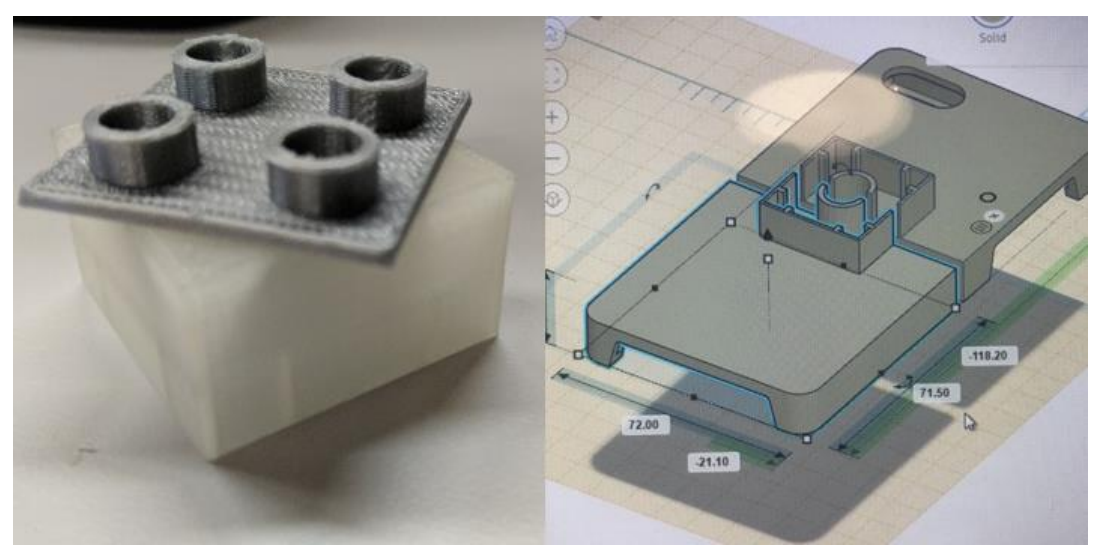

Figure 6 
Chess Set Team Challenge: Students were put into groups and given the task of developing a themed chess set. Teams were encouraged to get inspiration from existing figurine designs going all the way back to prehistoric times 35,000 years ago. The teams competed against each other to design the most aesthetically pleasing chess set, an example of some pieces is shown in Figure 7.

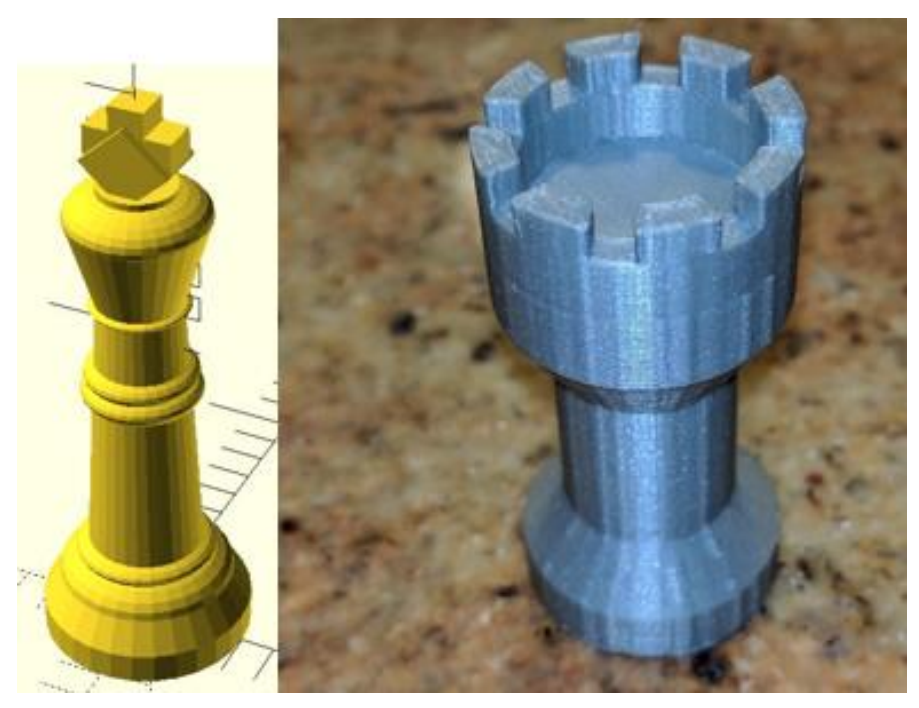

Figure 7

Fractal Geometry: "Why is geometry often described as "cold" and "dry"? One reason lies in its inability to describe the shape of a cloud, a mountain, a coastline, or a tree. Clouds are not spheres, mountains are not cones, coastlines are not circles, and bark is not smooth, nor does lightning travel in a straight line? Nature exhibits not simply a higher degree but an altogether different level of complexity." - Benoit Mandelbrot. This project highlighted the process of iteration and how it leads to "new" and complex fractal geometries. Fractal objects found in nature were examined and the mathematics used to recreate some of them in digital form was taught in class. This project was also intended to show students how to write code to describe recursively-defined objects. Figure 8 shows examples of two student's fractal objects; the Sierpinski Tetrahedron and the Menger Sponge.

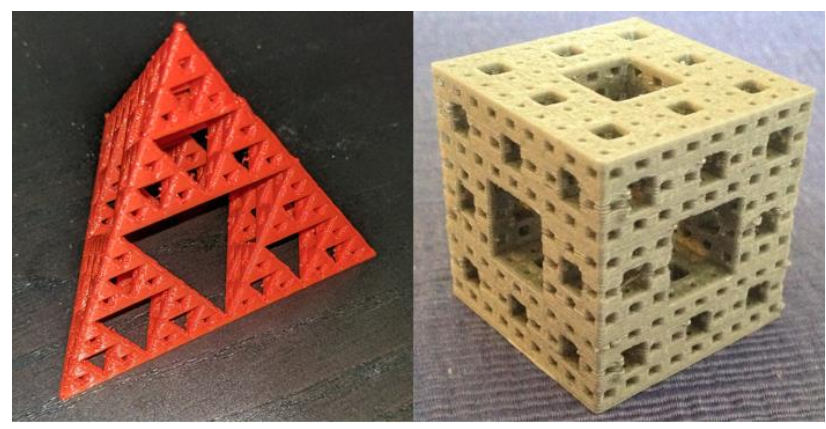

Figure 8 
Metallic Pendant: Not only can 3D printing create objects out of different types of plastic but it may also be used to directly print things made entirely out of metal. Students designed a pendant with the specifications required so that it may be printed out of metal. Students who wanted to pay for the metallic version of their pendant were able to do so by sending their model to be professionally printed through an online service. Figure 9 shows an example of a plastic pendant on the left and a matte black steel one on the right.

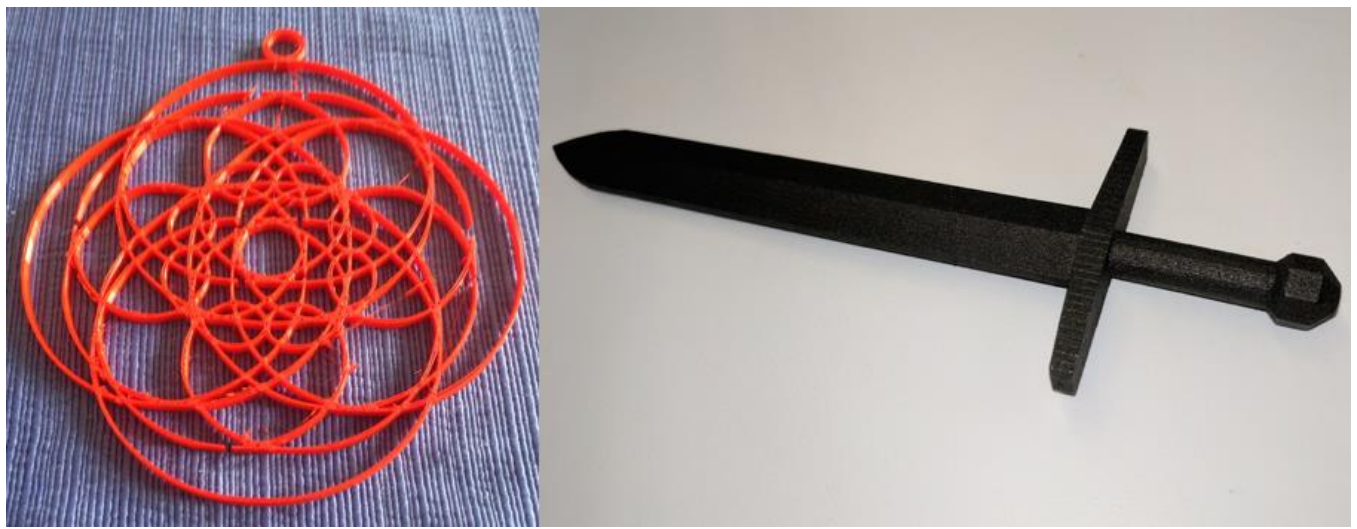

Figure 9

Dissolvable Support Required: Students learned throughout the semester, when designing an object, they need to pay particular attention to the complexity of the support it will require during the 3D printing process. For this project water soluble support material was used that allowed students to design without the limitations that usually accompany FDM printing. To that end, the design was up to the student, but it needed to not only benefit from but require dissolvable support for it to successfully print. Figure 10 is an example of a sealed bearing fabricated with dissolvable supports.
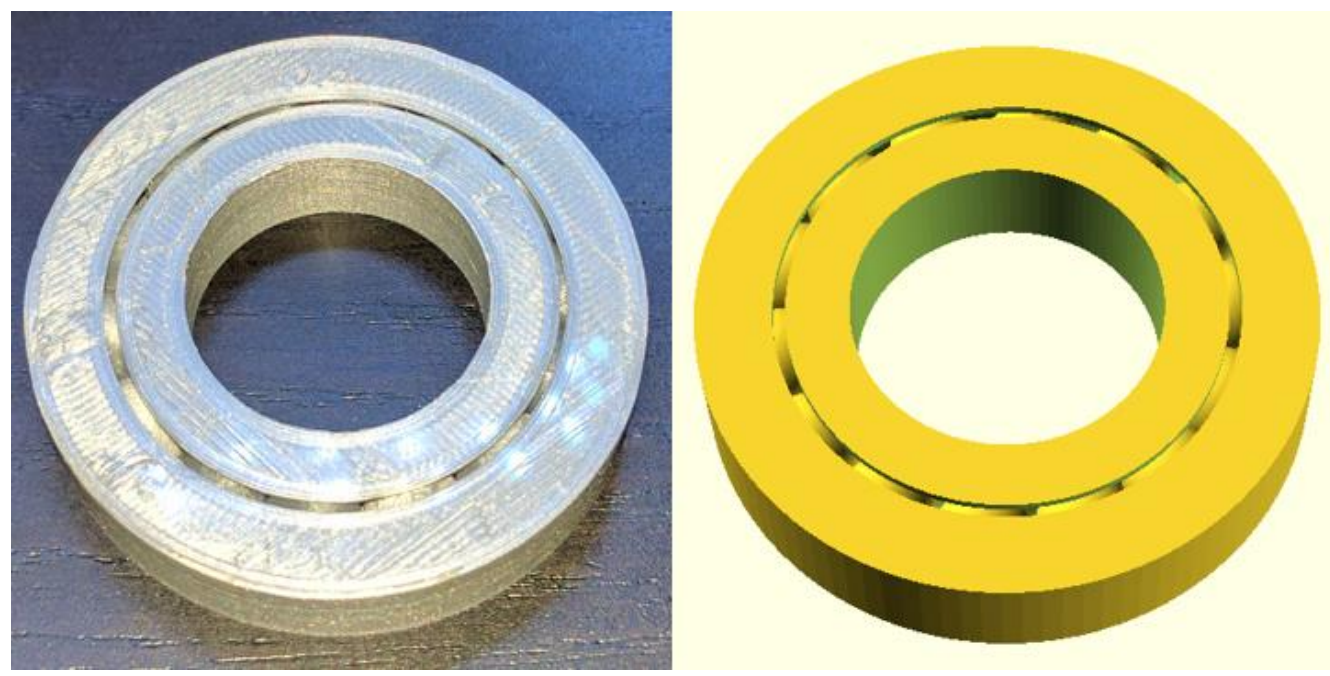

Figure 10 


\section{Customizable Object}

Creating an object with the end user in mind is a critical part of the design process. In this project students code their model in OpenSCAD to be fully customizable by the user. Figure 11 is an example of a few customizable objects fabricated by ET401 students.

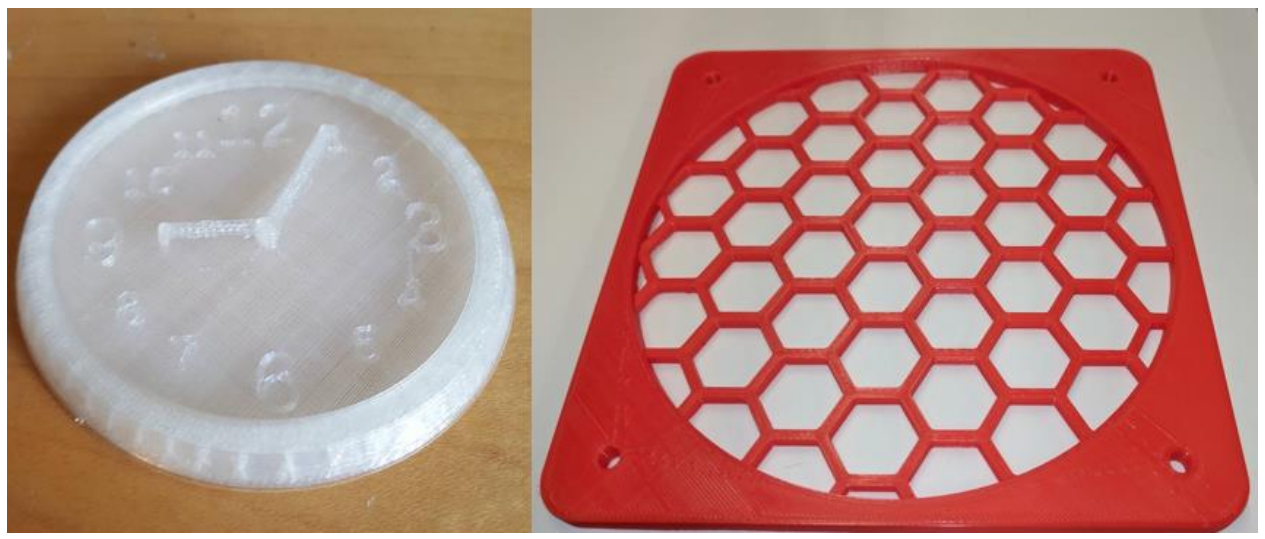

Figure 11

\section{Final Project: Action Figure}

The final project intended to combine all of the skills that students have learned throughout the course in a fun and creative design. Each student was to create a poseable action figure that meets the following conditions:

- The figure must make use of the dual extrusion capabilities of an Ultimaker 3 3D printer to produce a model with multiple colors.

- The figure must make use of movable components that involve either a ball and socket joint or a working hinge.

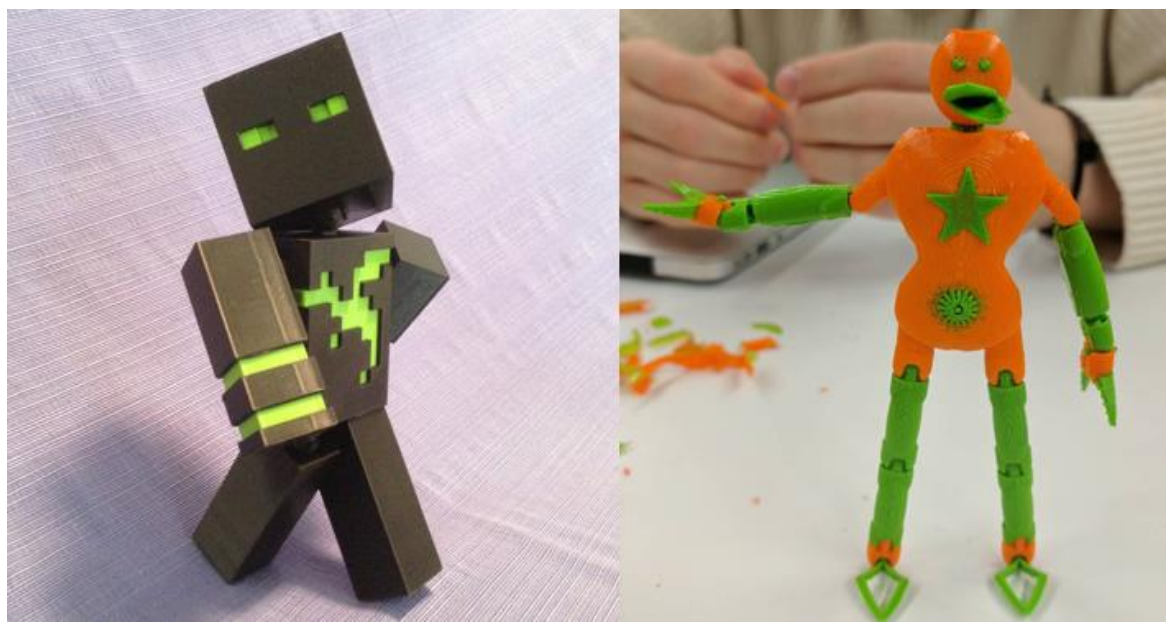

Figure 12

Figure 12 shows two examples of action figures designed and fabricated by ET401 students. In some cases students managed to get multiple moving components, in other cases students opted to pursue more aesthetic implementations. 


\section{Conclusion}

The UNHM-ET program has developed a course, ET401, in additive manufacturing with the purpose of getting both non-ET and ET majors to understand the impacts 3D printing will have on modern culture. During this work the ET 3D printing facility was made available to non-ET students taking the course. The course is intended to meet the University's Discovery program Environment-Technology-Society requirements. This course does not cover any ET program requirements but ET students are encouraged to take it for the opportunity to collaborate with other disciplines. The structure of the course and the assessment methods were described. The class has run for one semester and is currently running in the spring 2018 semester. The course will continue to be offered and as the class hits the enrollment cap additional sections may be added. For the future the ET program is purchasing a Laser Carver for the laboratory and will upgrade the printers as new models and innovations become available.

\section{References}

1. The University of New Hampshire Discovery program, https://www.unh.edu/discovery

2. Raleigh, M., Not a Household Name Students can't aspire to be engineers if they don't know the meaning of the term. PRISM American Society of Engineering Education January 2013

Available online: http://www.prism-magazine.org/jan13/frontiers.cfm

3. Bal, M., Abatan, A., (June 2017) Developing Additive Manufacturing Laboratory to Support Instruction and Research in Engineering Technology Paper Presented at the 2017 ASEE Annual Conference and Exposition, Columbus Ohio

4. Laura Taalman; http://makerhome.blogspot.com/2014/07/day-313-designing-penny-trapwith.html 